\title{
Métodos Estatísticos Mais Recorrentes nas Dissertações do Programa de Pós-Graduação em Ciências Contábeis da FURB
}

\author{
Statistical Methods More Applicants in Dissertations of \\ Graduate Program in Accounting from FURB \\ Lara Fabiana Dallabona \\ Mestranda do Programa de Pós Graduação em Ciências Contábeis (FURB) \\ Sabrina Nascimento \\ Ciências Contábeis (FURB)
}

Mestranda do Programa de Pós Graduação em

\section{Resumo}

O estudo buscou analisar os métodos estatísticos aplicados nas dissertações do programa de pós-graduação em ciências contábeis da Universidade Regional de Blumenau (FURB), no período de 2005 a 2009. A pesquisa de cunho descritivo foi realizada por meio de uma pesquisa documental e bibliográfica com abordagem quantitativa. A amostra compreendeu as 102 dissertações aprovadas e defendidas no programa de pós-graduação em ciências contábeis da FURB no período de 2005 a 2009. Os resultados demonstram que $43 \%$ das dissertações possuem natureza qualitativa e 69 dissertações pertencem à linha de Controle de Gestão. Nos recursos ilustrativos têm-se o predomínio de figuras, quadros e tabelas em $42 \%$ das dissertações analisadas, onde as hipóteses de pesquisa foram encontradas em 22 dissertações. Detectou-se uma evolução nos métodos estatísticos utilizados nos últimos anos, onde os mais recorrentes foram à análise multivariada dos dados (18\%), inferências estatísticas (15\%), regressão e correlação (10\%). A evolução da utilização de ferramentas estatísticas nas dissertações confeccionadas no programa de pós-graduação em ciências contábeis nos últimos anos tem como intuito o incentivo as pesquisas quantitativas, pois os métodos estatísticos ajudam no desenvolvimento das pesquisas realizadas e contribuem para a interpretação dos fenômenos sociais investigados a fim de proporcionar uma evolução científica.

Palavras-Chave: Métodos Estatísticos. Dissertações. Programa de Pós-Graduação em Ciências Contábeis.

\begin{abstract}
The study sought to analyze the statistical methods applied in the dissertations of the graduate program in accounting sciences from the Regional University of Blumenau (FURB) in the period 2005 to 2009. The descriptive research was conducted through a desk research and literature with a quantitative approach. The sample comprised the 102 dissertations approved and defended in the graduate program in accounting sciences FURB the period 2005 to 2009. The results show that 43\% of dissertations have qualitative dissertations and 69 belong to the line of Management Control. In the illustrative features are the predominance of figures and tables in $42 \%$ of the dissertations analyzed, where the research hypotheses were found in 22 dissertations. There was an evolution in the statistical methods used in recent years, where most applicants were to multivariate data analysis (18\%), statistical inferences (15\%), regression and correlation (10\%). The evolution of the use of statistical tools in the dissertations prepared at the graduate program in accounting sciences in recent years has the intention to encourage the quantitative research, because the statistical methods help in the development of research conducted and contribute to the interpretation of social phenomena investigated to provide a scientific developments.
\end{abstract}


Keywords: Statistical Methods. Dissertations. Graduate Program in Accounting.

\section{INTRODUÇÃO}

Os estudos relacionados à produção científica em contabilidade no Brasil, embora em pequenos números, tem sido desenvolvido em maior quantidade nos últimos anos. Os trabalhos encontrados são orientados pela bibliometria, consistindo na avaliação quantitativa de artigos, relatórios científicos e outros (THEÓPHILO; IUDICÍBUS, 2005). Gallon et al. (2008, p. 2) afirmam que "diversos estudos desenvolvidos até o momento tiveram como foco de investigação a qualidade e o rigor científico, o conteúdo e a forma, as estratégias metodológicas, a autoria e as referências bibliográficas da produção científica da área de Contabilidade brasileira".

As dissertações assim como outros estudos científicos passam por varias fases até sua elaboração final. Essa trajetória engloba o assunto a ser perseguido; delimitação do tema; identificação do objeto de investigação e dos objetivos; definição dos métodos e procedimentos de investigação; construção do marco teórico referencial; e coleta e análise dos dados (BEUREN, 2008). As tipologias de pesquisas relacionadas à abordagem do problema englobam duas metodologias: pesquisas qualitativas e pesquisas quantitativas. $\mathrm{Na}$ pesquisa qualitativa aplicam-se analises mais profundas relacionadas ao fenômeno estudado. E nas pesquisas quantitativas, caracterizam-se pelo emprego de instrumentos estatísticos, no que tange a coleta e tratamento dos dados (RAUPP; BEUREN, 2008).

Caneca e Vasconcelos $(2007$, p. 2) relatam que a "estatística pode ser definida como a parte da matemática aplicada que fornece métodos para a coleta, organização, descrição, análise e interpretação de dados". Os autores afirmam ainda que, "as informações estatísticas são concisas, específicas, eficazes e, quando analisadas com a ajuda de técnicas formais de análise estatística, fornecem subsídios imprescindíveis para as tomadas racionais de decisão".

As dissertações são estudos científicos elaborados ao final dos cursos stricto sensu para obtenção do título de mestre. O programa de pós-graduação em ciências contábeis da Universidade Regional de Blumenau (FURB) teve seu mestrado em ciências contábeis reconhecido pelo CEE/SC - Conselho Estadual de Educação de Santa Catarina, conforme Resolução $\mathrm{n}^{\mathrm{o}}$ 038, de 29 de junho de 2004 e recomendado pela Coordenação de Aperfeiçoamento de Pessoal de Nível Superior (CAPES) em 24 de maio 2005 (FURB, 2010).

De acordo com a CAPES (2010) o Brasil possui 24 programas de pós-graduação em contabilidade, sendo que destes programas quatro possuem os cursos mestrado e doutorado simultaneamente. Com a inclusão de novos cursos stricto sensu na área de ciências contábeis, e o aumento significante de instituições de ensino superior relacionado a essa área do saber, a produção científica em contabilidade teve um aumento significante nos últimos anos. Muitas vezes esse crescimento não é acompanhado pelo devido rigor teórico, comprometendo em algumas situações, a qualidade dos trabalhos científicos que estão sendo desenvolvidos (GALLON, et al., 2008).

Com vistas a contribuir para esta discussão no que tange aos métodos estatísticos que estão sendo utilizados nas dissertações dos programas de pós-graduação em ciências contábeis, o estudo pretende responder a indagação que norteia a presente pesquisa: Quais são os métodos quantitativos mais recorrentes nas dissertações do programa de pós-graduação em ciências contábeis da Universidade Regional de Blumenau? Assim, têm-se como objetivo principal analisar os métodos estatísticos aplicados nas dissertações do programa de pósgraduação em ciências contábeis da Universidade Regional de Blumenau, no período de 2005 a 2009. Os objetivos específicos buscaram o que segue: 1) caracterização das dissertações veiculadas no programa de pós-graduação analisado; 2) linhas de pesquisa que abrangem as dissertações estudadas; 3) tipologia de pesquisa quanto aos objetivos, hipóteses e recursos 
ilustrativos das dissertações analisadas; e 4) análise dos métodos estatísticos aplicados nas dissertações estudadas.

Justifica-se a relevância desta pesquisa pelo embasamento dos métodos quantitativos que envolvem a produção científica das dissertações de mestrado em ciências contábeis da Universidade Regional de Blumenau. A contribuição envolve fatores relacionados à melhoria da qualidade da pesquisa na área contábil, analisando a possibilidade do presente estudo atribuir aos demais programas de pós-graduação stricto sensu em ciências contábeis subsídios importantes para elaboração de pesquisas científicas (SANTOS; KLANN; RAUSCH, 2010).

O presente estudo está estruturado em sete seções, iniciando com esta de caráter introdutório. Na sequência faz-se uma incursão teórica sobre métodos quantitativos e a contabilometria na contabilidade, além de estudos anteriores que objetivaram analisar teses e dissertações. Em seguida, apresenta-se a metodologia empregada para consecução da presente pesquisa seguida da análise dos dados. Ao final, demonstram-se as considerações finais e tece recomendações para futuras pesquisas.

\section{MÉTODOS QUANTITATIVOS E CONTABILOMETRIA NA CONTABILIDADE}

A utilização de métodos quantitativos no tratamento de problemas relacionados a pesquisas em contabilidade no Brasil é relativamente recente. Entretanto em países como os Estados Unidos, observam-se vários periódicos de contabilidade com artigos publicados que usam estatísticas avançadas (RAUPP; BEUREN, 2008). Atualmente os métodos quantitativos relacionados à estatística estão sendo aplicados em vários estudos científicos, sobretudo no campo da Contabilidade.

Caneca e Vasconcelos (2007, p. 2) afirmam que "a estatística pode ser definida como a parte da matemática aplicada que fornece métodos para a coleta, organização, descrição, análise e interpretação de dados". Os autores relatam ainda que "as informações estatísticas são concisas, específicas, eficazes e, quando analisadas com a ajuda de técnicas formais de análise estatística, fornecem subsídios imprescindíveis para as tomadas racionais de decisão".

Dessa forma, a contabilidade tem utilizado os métodos quantitativos por meio das técnicas estatísticas para a tomada de decisão. Figueiredo e Moura $(2001$, p. 1) asseveram que "a utilização de modelos contábeis baseados em métodos quantitativos tem se tornado cada vez mais freqüente decorrente do rápido desenvolvimento da tecnologia da informação e da utilização corriqueira dos microcomputadores". Neste contexto, Figueiredo e Moura (2001, p. 2) destacam que "é tarefa dos contadores transformar dados em informações, pois, os dados são simplesmente um conjunto de fatos expressos sob a forma de símbolos ou caracteres, incapazes de influenciar decisões até serem transformados em informações".

Iudícibus (1982) apud Matsumoto, Pereira e Nascimento (2006) relatam que a Contabilometria vem sendo inserida como uma nova área do conhecimento contábil, que almeja desenvolver o raciocínio matemático dos contadores por meio da aplicação de métodos quantitativos na solução de seus problemas contábeis. Configurando-se como desafio da contabilidade para produzir informações relevantes ao processo de gestão. Silva, Chacon e Santos (2005, p. 5) afirmam que "a contabilometria representa a utilização de metodologia científica de Métodos Quantitativos (Matemática, Estatística e Informática) na Contabilidade". Os autores asseveram ainda que a utilização da contabilometria implica na criação de cenários contábeis que por ventura venha a auxiliar o gestor no processo de tomada de decisão.

$\mathrm{O}$ ensino da contabilometria vem sendo praticado por algumas instituições de ensino, tais como: Universidade de São Paulo (USP); Universidade Regional Integrada do Alto Uruguai e das Missões (URI); Faculdade de Boa Viagem - Recife/PE. Nos objetivos e programas dessa disciplina de contabilometria, há a preocupação da utilização de ferramentas 
estatísticas (regressão linear, métodos dos mínimos quadrados, etc.), matemática (programação linear, etc.) e de informática (resolução de problemas pelo Microsoft Excel, etc.) que proporcionem soluções a problemas gerenciais, de planejamentos, de controles e tomadas de decisão no âmbito da gestão econômico-financeiro de empresas (SILVA; CHACON; SANTOS, 2005). Na contabilometria, o aluno precisa compreender o objetivo dos métodos quantitativos, conhecendo a definição dos principais termos que são utilizados, bem como ter uma visão geral da forma que estes operam algoritmicamente, verificando como podem ser resolvidos por meio de uma ferramenta computacional e por último não menos importante como podem ser interpretados os seus resultados (NOSSA; GARCIA, 2002). Neste contexto, percebe-se a necessidade de estudar os métodos estatísticos mais recorrentes na literatura como: estatísticas descritivas, inferências estatísticas, regressão e correlação, análise multivariada dos dados, abordagem multicritério, modelagem matemática, metaheurística e Data mining, sendo explicitados à luz dos autores clássicos que permeiam a literatura vigente como Spiegel (1993), Maroco (2003) e Corrar, Paulo e Dias Filho (2007) com as devidas descrições destes métodos, conforme explicitado no Quadro 1.

\begin{tabular}{|c|c|}
\hline $\begin{array}{c}\text { Ferramentas } \\
\text { Estatísticas }\end{array}$ & Descrição / Conceito \\
\hline $\begin{array}{l}\text { Estatística } \\
\text { Descritiva }\end{array}$ & $\begin{array}{l}\text { Maroco (2003) elenca como estatística descritiva, as medidas de tendência central; } \\
\text { medidas de dispersão; medidas de assimetria e achatamento; medidas de associação e } \\
\text { representação gráfica de resultados. As medidas de tendência central procuram caracterizar } \\
\text { os valores das variáveis que ocorrem com maior freqüência. Nessas medidas as estatísticas } \\
\text { que são utilizadas com maior freqüência são: média, mediana e moda. Nas medidas de } \\
\text { dispersão, as estatísticas mais utilizadas, de acordo com autor são: o coeficiente de } \\
\text { variação; o intervalo de variação e a amplitude inter-quartis. No que tange as medidas de } \\
\text { assimetria e achatamento. Maroco (2003, p. 30) relata que "caracterizam a forma da } \\
\text { distribuição dos elementos da população amostrados em torno da média. A Assimetria de } \\
\text { uma distribuição pode ser caracterizada pelo enviesamento (skewness) que essa } \\
\text { distribuição apresenta relativamente à média". A representação gráfica de resultados } \\
\text { objetiva visualizar as características das variáveis em estudo, apresentando os resultados } \\
\text { de forma simples e de fácil memorização. Os gráficos podem ser representados por } \\
\text { histogramas de freqüências, gráficos de barras, gráficos circulares diagramas de dispersão } \\
\text { e outros (MAROCO, 2003). }\end{array}$ \\
\hline $\begin{array}{l}\text { Inferências } \\
\text { Estatísticas }\end{array}$ & $\begin{array}{l}\text { A inferência estatística aborda algumas funções de densidade de probabilidade que são } \\
\text { utilizadas com maior freqüência em análise estatísticas. As funções são: distribuição } \\
\text { normal; distribuição do Qui-quadrado }\left(\mathrm{x}^{2}\right) \text {; distribuição t-Student; Distribuição F- } \\
\text { Snedecor; Distribuição Binomial. (MAROCO, 2003). }\end{array}$ \\
\hline $\begin{array}{l}\text { Regressão e } \\
\text { Correlação }\end{array}$ & $\begin{array}{l}\text { Na teoria da correlação, Spiegel (1993, p. 359) afirma que "quando estão no jogo somente } \\
\text { duas variáveis, fala-se em correlação e regressão simples. Quando se trata de mais de } \\
\text { duas variáveis, fala-se de correlação e regressão múltiplia". No que tange a teoria da } \\
\text { correlação, as técnicas estatísticas utilizadas são: correlação e regressão; correlação linear; } \\
\text { medidas de correlação, linha de regressão de mínimos quadrados; erro padrão da } \\
\text { estimativa; variação explicada e não-explicada; coeficiente de correlação; observações } \\
\text { sobre o coeficiente de correlação; fórmula de covariância para o coeficiente de correlação } \\
\text { linear; correlação ordinal; correlação de séries temporais; correlação de atributos; teoria } \\
\text { amostral da correlação. Teoria amostral da regressão; correlação múltipla (SPIEGEL, } \\
\text { 1993, p. X). }\end{array}$ \\
\hline $\begin{array}{l}\text { Análise } \\
\text { Multivariada } \\
\text { dos Dados }\end{array}$ & $\begin{array}{l}\text { Conforme Corrar, Paulo e Dias Filho }(2007, \text { p. 2) "a análise multivariada refere-se a um } \\
\text { conjunto de métodos estatísticos que torna possível a análise simultânea de medidas } \\
\text { múltiplas para cada indivíduo, objeto ou fenômeno estudado". } \\
\text { Esta análise está relacionada a todos os métodos estatísticos no qual realizam estudo } \\
\text { estatístico envolvendo múltiplas variáveis em um único relacionamento ou até mesmo } \\
\text { conjunto de relações (CORRAR, PAULO E DIAS FILHO, 2007). As técnicas de análise } \\
\text { multivariada discutidas e abordadas por Corrar, Paulo e Dias filho (2007) são: regressão } \\
\text { múltipla; análise discriminante; regressão logística; análise fatorial; análise de } \\
\text { conglomerados (clusters analysis); MDS (escalonamento multidimensional); redes } \\
\text { neurais; lógica nebulosa. }\end{array}$ \\
\hline
\end{tabular}




\begin{tabular}{|c|l|}
\hline $\begin{array}{c}\text { Abordagem } \\
\text { multicritério }\end{array}$ & $\begin{array}{l}\text { A abordagem multicritério envolve entre outros, as análises envoltórias de dados, bem } \\
\text { como análise hierárquica de processos (HPA). Para Lyra (2008, p. 54) "o método AHP } \\
\text { compara um elemento do nível hierárquico mais alto com todos os elementos do nível } \\
\text { imediatamente inferior". }\end{array}$ \\
\hline $\begin{array}{c}\text { Modelagem } \\
\text { Matemática }\end{array}$ & Está relacionada a diversos cálculos matemáticos. \\
\hline Metaheurística & $\begin{array}{l}\text { A estatística focada na metaheurística está relacionada aos conjuntos difusos. Fassina } \\
\text { (2006, p. 83) relata que "a construção do modelo especialista difuso compreende três } \\
\text { fases: a) construção das variáveis difusas de entrada, chamadas de fuzzificação; b) } \\
\text { construção das regras de inferência pertinentes ao modelo; e, c) construção das variáveis } \\
\text { de saída, chamadas de defuzzificação". }\end{array}$ \\
\hline Data Mining & $\begin{array}{l}\text { No que tange ao método estatístico Data Mining, Santos et al. (2007) apud Grilo Júnior et } \\
\text { al. (2009, p. 7) relatam que "o Data Mining (mineração de dados) é um processo de } \\
\text { extração de dados em DW objetivando a descoberta de conhecimento "escondido", tendo } \\
\text { como entrada o dado em estado bruto (ainda não trabalhado), além disso, deve criar } \\
\text { modelos que possam ser utilizados na tomada de decisão". }\end{array}$ \\
\hline
\end{tabular}

Quadro 1 - métodos estatísticos mais recorrentes na literatura

O Quadro 1 demonstra-se a descrição dos métodos estatísticos mais utilizados de acordo com os autores clássicos, porém reserva-se a conceituá-las de forma breve sem a pretensão de esgotar o tema.

\section{ESTUDOS ANTERIORES}

Nesta seção, apresentam-se algumas pesquisas veiculadas em anais de congressos e periódicos científicos, tais como: Encontro Nacional da Associação dos Programas de PósGraduação em Administração (EnANPAD), Seminário Nacional de Bibliotecas Universitárias, Revista Vista \& Revidas e Cadernos de Estudos, além de algumas teses e dissertações que versam sobre a análise de teses e dissertações dos programas de pósgraduação realizadas por Riccio, Sakata e Carastan (1999), Theóphilo e Iudícibus (2005), Ribeiro Filho et al. (2007), Galvino e Souza (2008) e Santos, Klann e Rausch (2010).

Riccio, Sakata e Carastan (1999) buscaram analisar as tendências seguidas de Contabilidade em pesquisa no Brasil a partir do primeiro trabalho até hoje (1999). Nesta pesquisa os autores analisaram a distribuição, as características e a evolução dos textos acadêmicos de contabilidade produzidos nas Universidades Brasileiras entre 1962 e 1999. Incluindo ainda todas as dissertações e teses geradas em programas oficiais de Pós-Graduação strictu sensu em contabilidade. A análise focou as tendências quanto ao número de dissertações e teses por ano, ao método de pesquisa empregado e à variação temática coberta nos campos de pesquisa em contabilidade. O estudo é importante para o desenvolvimento contínuo da educação de Contabilidade, bem como harmonização contábil.

No entanto, Theóphilo e Iudícibus (2005) tiveram como foca a produção científica em Contabilidade no Brasil sob uma ótica ainda menos explorada: a da Epistemologia. Essa pesquisa analisou 238 trabalhos, publicados no período de 1994 a 2003, sendo 178 teses e dissertações dos programas de pós-graduação recomendados pela Coordenação de Aperfeiçoamento de Pessoal de Nível Superior (CAPES); 26 artigos de periódico e 34 trabalhos de congresso com melhores avaliações contempladas pelo sistema Qualis/CAPES. Os resultados da análise e a discussão crítica desenvolvidas atestam uma melhoria geral na qualidade metodológica dos trabalhos ao longo do período estudado.

Ribeiro Filho et al. (2007) almejam analisar a partir das abordagens metodológicas, a problemática das dissertações aprovadas no programa de mestrado Multiinstitucional e InterRegional de Pós-Graduação em Ciências Contábeis do Convênio da Universidade de Brasília (UnB), Universidade Federal da Paraíba (UFPB), Universidade Federal de Pernambuco (UFPE) e Universidade Federal de Natal (UFRN). Neste trabalho foram analisadas 54 
dissertações sob diversas perspectivas, onde se pode constatar que existe a necessidade de um aprimoramento na formação básica do pesquisador, quanto ao aprofundamento de conhecimento sobre metodologia científica, no que tange a ampliar os conhecimentos relacionados com os fundamentos filosóficos e epistemológicos da pesquisa, assim como no às técnicas de investigação.

Galvino e Souza (2008) analisaram por meio das referências bibliográficas até que ponto o acervo das bibliotecas tem atendido as necessidades informacionais presentes nas citações dos mestrandos. Os autores realizaram um estudo bibliométrico, por meio da análise das referências bibliográficas das dissertações do Programa de Pós-Graduação em Psicologia Social do Centro de Ciências Humanas, Letras e Artes da Universidade Federal da Paraíba (UFPB). Percebe-se que $80 \%$ dos títulos citados nas dissertações, confirmam a missão das bibliotecas da UFPB de dar suporte informacional aos programas de ensino, pesquisa e extensão. E as referências que não foram encontrados no acervo, indicam que as bibliotecas universitárias precisam estar numa constante renovação de seus títulos.

E por fim, Santos, Klann e Rausch (2010) traçaram o perfil das dissertações defendidas no âmbito dos Programas de Pós-Graduação em Ciências Contábeis da Universidade de São Paulo (USP) e da Universidade Regional de Blumenau (FURB). Nesta pesquisa foram analisadas 66 dissertações defendidas no período de 2007 a 2009, sendo 35 da FEA/USP e 31 da FURB. As dissertações foram analisadas sobre quatro aspectos: demografia de autoria; estrutura; formas de argumentações e referências. Em relação à demografia dos autores, constatou-se que houve predominância do sexo masculino nos dois programas investigados. Percebeu-se também que houve o predomínio de um ou dois professores nas orientações no período analisado em ambas as instituições. Verificou-se que a FEA/USP concentrou suas pesquisas na área de Contabilidade Financeira, ao passo que a FURB esteve mais voltada à área de Contabilidade Gerencial. Quanto à estrutura das dissertações, constatou-se a predominância de estudos baseados em pesquisa documental e de levantamento em ambos os programas. Em termos de análise das referências, concluiu-se que a FEA/USP apresentou-se um pouco mais madura na utilização de fontes de informação, prevalecendo periódicos ao invés de livros, além de referenciar mais fontes internacionais.

\section{METODOLOGIA DE PESQUISA}

A pesquisa caracteriza-se como descritiva, pois tem como objetivo identificar os métodos quantitativos aplicados nas dissertações do programa de pós-graduação em ciências contábeis da Universidade Regional de Blumenau, no período de 2005 a 2009. Cervo e Bervian (1996, p. 49) destacam que a pesquisa descritiva "trata-se do estudo e da descrição das características, propriedades ou relações existentes na comunidade, grupo ou realidade pesquisada". Para tanto, na estratégia de pesquisa optou-se por uma pesquisa bibliográfica que conforme Martins e Theóphilo (2007, p. 54) visam "explicar e discutir um assunto, tema ou problema com base em referências publicadas em livros, periódicos, revistas, enciclopédias, dicionários, jornais, sites, CDs, anais de congressos etc.".

Quanto ao problema, o estudo apresenta uma abordagem quantitativa ao identificar e quantificar os métodos quantitativos mais recorrentes utilizados nas dissertações do programa de pós-graduação em ciências contábeis da FURB. Para Boudon (1989, p. 24) "as pesquisas quantitativas podem ser definidas como as que permitem recolher, num conjunto de elementos, informações comparáveis entre um elemento e outro". Cabe mencionar ainda, que a população deste estudo compreende as dissertações aprovadas e defendidas no programa de pós-graduação em ciências contábeis da Universidade Regional de Blumenau, no período de 2005 a 2009, sendo a amostra contemplada pelas 102 dissertações disponíveis no sítio do programa e coletadas durante o mês de maio de 2010, conforme dispostas no Quadro 2. 


\begin{tabular}{|c|c|c|}
\hline Ano & $\begin{array}{c}\mathbf{N}^{0} . \\
\text { dissertações }\end{array}$ & Autores \\
\hline 2005 & 47 & $\begin{array}{l}\text { Abrao Fiorese; Adalberto Andreatta; Ademir Proner; Amilton Fernando Cardoso; } \\
\text { Carlos Fabiano Fistarol; Célio Corrêa dos Santos; Celso da Rosa Filho; Celso Galante; } \\
\text { Cleunice Fátima Frozza; Darcle Costa Silva Haussmann; Délci Tamanini; Edison Luiz } \\
\text { de Oliveira; Eli Teresinha Biscaro; Elizabeth Krummenacher Marçal; Francisco } \\
\text { Felippini; Gilson Luiz Leidens; Gilvanio Ballmann; Iara Regina dos Santos Parisotto; } \\
\text { Ildo Fabris; Isair Sell; Ivanete Linke; João Francisco Morozini; Joarês Tártari; Joselito } \\
\text { Mariotto; Juceli Antonio Biazzi; Júlio Alberto Wickert; Luiz Felipe Haider; Luiz } \\
\text { Roberto Romanowski; Manoel Salésio Mattos; Marcos Moreira Pinto; Maria } \\
\text { Clementina Bruço Ostrowski; Miriam Leopoldina Herbst de Lima; Omeri Dedonatto; } \\
\text { Pablo Rozano Winckler; Paulo Onildo de Matos; Paulo Oscar Gielow; Paulo Roberto } \\
\text { da Cunha; Renato Luis Artifon; Rute Cristina Meurer Soares; Sady Mazzioni; Sigolf } \\
\text { Ulrich; Suzete Antonioeta Lizote; Tarcísio Pedro da Silva; Valdecir Knuth; Valmir } \\
\text { Alberto Thome; Vander da Silveira Melo. }\end{array}$ \\
\hline 2006 & 21 & $\begin{array}{l}\text { Aldecir José Theodoro; Alessandra Vasconcelos Gallon; Aliciane Aparecida Novello; } \\
\text { Aloisio Grunow; Anderson Léo Sabadin; Antonio de Oliveira; Dulcicléa de Jesus } \\
\text { Palha; Emerson Hochsteiner de Vasconcelos; Franciane Luiza Salamoni; Ieda } \\
\text { Margarete Oro; Ivan Taciano Goede; Jorge Eidt; Loreni Maria dos Santos Braum; } \\
\text { Marcia Batista Pereira; Marcio Alves; Marcos Aurélio Custódio; Marli Terezinha } \\
\text { Vieira; Paulo Henrique Fassina; Paulo Roberto Elias; Rosenei Novochadlo da Costa; } \\
\text { Valdenir Menegat. }\end{array}$ \\
\hline 2007 & 12 & $\begin{array}{l}\text { Adilson Costa; Antônio Carlos Schlindwein; Celia de Souza; Dino Luiz Pasqual; Édina } \\
\text { Elisangela Zellmer Fietz; Elza Terezinha Cordeiro Müller; Fabio Darci Kowalski; José } \\
\text { Carlos de Souza; Marines Lucia Boff; Marino Luiz Eyerkaufer; Reinaldo de Lima } \\
\text { Junior; Roberto Carlos Klann. }\end{array}$ \\
\hline 2008 & 15 & $\begin{array}{l}\text { Adriano José da Silva; Adriano Lourensi; Carlos Alexandre G. Costa; Eder Paulo da } \\
\text { Silva; Gilton Paulo da Silva; Jefferson Fernando Grande; Juliana Pinto; Julien Ariani } \\
\text { de Souza Laudelino; Josiane Carla Jamoski Luciani; Luciano Fernandes; Nadia Mar } \\
\text { Bogoni; Noemia Schroeder Althoff; Ricardo Marsilio Stedile; Sidiney Rodrigues; } \\
\text { Vinicius Costa da Silva Zonatto. }\end{array}$ \\
\hline 2009 & 7 & $\begin{array}{l}\text { Adriana Kroenke; Alfredo Ernesto Erfurth; Aline Fernandes de Oliveira Czesnat; Ari } \\
\text { Sothe; Everaldo Leonel de Oliveira; Gilson Jober Fachini; Gisele Spessatto }\end{array}$ \\
\hline
\end{tabular}

Quadro 2 - Dissertações do programa de pós-graduação em ciências contábeis defendidas no período de 2005 a 2009

Fonte: Dados da pesquisa.

$\mathrm{Na}$ fase de coleta dos dados, após a coleta das 102 dissertações que compõem a amostra, foi realizada a leitura do resumo, metodologia e técnicas de pesquisa, bem como a análise dos resultados para atingir o objetivo do estudo que é identificar os métodos quantitativos aplicados nas dissertações. A fim de facilitar este processo, utilizou-se uma ficha padronizada por meio de uma planilha eletrônica do Microsoft Excel que contemplava os seguintes itens: a) autor; b) ano de defesa; c) título; d) objetivo geral; e) tipologia de pesquisa quanto à abordagem do problema; f) população e amostra; g) ferramenta estatística; h) procedimentos estatísticos; i) tipologia de pesquisa quanto aos procedimentos; j) instrumento de coleta de dados; k) limitações da pesquisa; 1) tratamento e análise dos dados; m) apresentação dos resultados; n) hipóteses da pesquisa; o) linhas de pesquisa; e p) orientadores. Em relação aos procedimentos adotados na análise quantitativa dos dados, utilizou-se da estatística descritiva.

\section{ANÁLISE DOS DADOS}

$\mathrm{Na}$ descrição e análise dos dados coletados evidenciam-se a caracterização das dissertações veiculadas no programa de pós-graduação analisado; as linhas de pesquisa que abrangem as dissertações estudadas; as tipologias de pesquisa quanto aos objetivos, hipóteses 
e recursos ilustrativos das dissertações analisadas; bem como a análise dos métodos estatísticos aplicados nas dissertações estudadas.

\subsection{Caracterização das dissertações veiculadas no programa de pós-graduação analisado}

Neste item, buscou-se caracterizar as dissertações defendidas no programa de pósgraduação em ciências contábeis da FURB por meio da quantidade de dissertações aprovadas e defendidas por ano e relacioná-las ao gênero dos autores, conforme a Tabela 1.

Tabela 1 - Quantidade de dissertações defendidas em relação ao gênero dos autores

\begin{tabular}{l|c|c|c|c|c|c}
\hline \multirow{2}{*}{ Gênero } & \multicolumn{7}{c}{ Quantidade de dissertações defendidas por ano } \\
\cline { 2 - 7 } & $\mathbf{2 0 0 5}$ & $\mathbf{2 0 0 6}$ & $\mathbf{2 0 0 7}$ & $\mathbf{2 0 0 8}$ & $\mathbf{2 0 0 9}$ & Total \\
\hline Homens & 36 & 12 & 8 & 10 & 4 & 70 \\
\hline Mulheres & 11 & 9 & 4 & 5 & 3 & 32 \\
\hline Dissertações defendidas & 47 & 21 & 12 & 15 & 7 & 102 \\
\hline$\%$ Homem & $77 \%$ & $57 \%$ & $67 \%$ & $67 \%$ & $57 \%$ & - \\
\hline \% Mulher & $23 \%$ & $43 \%$ & $33 \%$ & $33 \%$ & $43 \%$ & - \\
\hline
\end{tabular}

Fonte: Dados da pesquisa.

Observa-se na Tabela 1 que há predominância do gênero masculino em relação ao gênero feminino. Para tanto, o ano de 2005 apresentou maior índice de dissertações compostas pelo gênero masculino (77\%) das dissertações defendidas. Em 2007 totalizou-se 12 dissertações, onde 8 eram do sexo masculino e 4 do sexo feminino. Percebe-se ainda que, em 2008 das 15 dissertações defendidas 10 eram homens e 5 mulheres. E por fim, o ano de 2009 apresentou um equilíbrio relativo entre os gêneros, onde $57 \%$ eram homens e $43 \%$ mulheres.

\subsection{Linhas de pesquisa que abrangem as dissertações estudadas}

Em relação às linhas de pesquisa do programa de pós-graduação, percebeu-se que todas as dissertações apresentaram no resumo a linha de pesquisa na qual estavam vinculadas, onde podemos observar os resultados dispostas na Tabela 2.

Tabela 2 - Linhas de pesquisa do programa de pós-graduação investigado

\begin{tabular}{l|c|c|c|c|c|c}
\hline \multirow{2}{*}{$\begin{array}{c}\text { Linhas de pesquisa do programa de pós- } \\
\text { graduação em ciências contábeis da FURB }\end{array}$} & \multicolumn{2}{c}{ Quantidade de dissertaçes defendidas por ano } \\
\cline { 2 - 8 } & $\mathbf{2 0 0 5}$ & $\mathbf{2 0 0 6}$ & $\mathbf{2 0 0 7}$ & $\mathbf{2 0 0 8}$ & $\mathbf{2 0 0 9}$ & Total \\
\hline Contabilidade Financeira & 17 & 8 & 4 & 2 & 2 & $\mathbf{3 3}$ \\
\hline Controle de Gestão & 30 & 13 & 8 & 13 & 5 & $\mathbf{6 9}$ \\
\hline
\end{tabular}

Fonte: Dados da pesquisa.

Constata-se na Tabela 2 que a linha de pesquisa dominante no programa de pósgraduação em ciências contábeis da FURB foi Controle de Gestão, onde das 102 dissertações defendidas e aprovadas, 69 pertencem à linha de pesquisa Controle de Gestão, e 33 dissertações pertencem à linha de pesquisa em Contabilidade Financeira.

\subsection{Tipologia de pesquisa quanto aos objetivos, recursos ilustrativos e hipóteses das dissertações analisadas}

Este item apresenta as tipologias de pesquisa adotada nas dissertações dos programas de pós-graduação em ciências contábeis da FURB, onde se analisa os objetivos destas dissertações quando aparecem de forma qualitativa, quantitativa e/ou qualitativa-quantitativa simultaneamente, em relação aos tipos de recursos ilustrativos utilizados e na quantificação do número de hipóteses utilizadas nas dissertações, conforme a Tabela 3. 
Tabela 3 - Classificação das dissertações quanto ao objetivo das pesquisas

\begin{tabular}{c|c|c|c}
\hline \multirow{2}{*}{ Ano de defesa } & \multicolumn{3}{|c}{ Tipologia de pesquisa quanto aos objetivos } \\
\cline { 2 - 4 } & Qualitativa & Quantitativa & Qualitativo-Quantitativa \\
\hline $\mathbf{2 0 0 5}$ & 22 & 17 & 8 \\
\hline $\mathbf{2 0 0 6}$ & 4 & 10 & 7 \\
\hline $\mathbf{2 0 0 7}$ & 1 & 7 & 4 \\
\hline $\mathbf{2 0 0 8}$ & 3 & 6 & - \\
\hline $\mathbf{2 0 0 9}$ & 3 & 4 & $\mathbf{2 5}$ \\
\hline Total & $\mathbf{3 3}$ & $\mathbf{4 4}$ & $\mathbf{2 5 \%}$ \\
\hline $\mathbf{( \% )}$ & $\mathbf{3 2 \%}$ & $\mathbf{4 3 \%}$ & \\
\hline
\end{tabular}

Fonte: Dados da pesquisa.

Percebe-se que na Tabela 3 que 43\% das dissertações apresentam natureza quantitativa em relação ao seu objetivo de pesquisa, sendo $32 \%$ de cunho qualitativo e $25 \%$ qualitativoquantitativa. No ano 2005, observou-se o predomínio das pesquisas qualitativas (22) quando comparada as 17 pesquisas de natureza quantitativa e oito qualitativo-quantitativa. Para tanto, em 2006, 2007 e 2009 constatou-se o predomínio das pesquisas quantitativas com 10, 7 e 4 dissertações respectivamente. Para tanto, em 2008 percebeu-se que ocorreu uma homogeneidade nas pesquisas de natureza quantitativa e qualitativo-quantitativa, pois ambas estavam presentes em seis dissertações respectivamente. Dessa forma, infere-se o predomínio da abordagem quantitativa nas dissertações veiculadas no programa de pós-graduação analisado que ressaltam a afirmação de Santos, Klann e Rausch (2010) quando relatam que esse predomínio nas pesquisas quantitativas, já era esperado, visto que as pesquisas quantitativas procuram quantificar determinado fenômeno utilizando diversos métodos estatísticos.

No que tange aos recursos ilustrativos utilizados nas dissertações, verifica-se o uso de gráficos, tabelas, quadros e figuras. Com vistas, a identificar estes recursos nas dissertações do programa de pós-graduação investigado, a Tabela 4 apresenta estes recursos ilustrativos.

Tabela 4 - Recursos ilustrativos utilizados nas dissertações do programa de pós-graduação analisado

\begin{tabular}{l|c|c|c|c|c|c|c}
\hline \multirow{2}{*}{$\begin{array}{c}\text { Categorização dos } \\
\text { Recursos ilustrativos }\end{array}$} & \multicolumn{7}{c}{ Quantidade de dissertações defendidas por ano } \\
\cline { 2 - 9 } & $\mathbf{2 0 0 5}$ & $\mathbf{2 0 0 6}$ & $\mathbf{2 0 0 7}$ & $\mathbf{2 0 0 8}$ & $\mathbf{2 0 0 9}$ & Total & $\mathbf{( \% )}$ \\
\hline Figuras & 1 & 1 & 0 & 0 & 0 & $\mathbf{2}$ & $\mathbf{2 \%}$ \\
\hline Figuras e tabelas & 2 & 1 & 1 & 0 & 0 & $\mathbf{4}$ & $\mathbf{4 \%}$ \\
\hline Figuras, quadros e tabelas & 15 & 9 & 10 & 6 & 3 & $\mathbf{4 3}$ & $\mathbf{4 2 \%}$ \\
\hline Figuras, gráficos e quadros & 2 & 0 & 0 & 1 & 0 & $\mathbf{3}$ & $\mathbf{3 \%}$ \\
\hline Figuras, gráficos e tabelas & 2 & 0 & 0 & 0 & 0 & $\mathbf{2}$ & $\mathbf{2 \%}$ \\
\hline Figuras, gráficos, quadros e tabelas & 9 & 8 & 0 & 1 & 0 & $\mathbf{1 8}$ & $\mathbf{1 8 \%}$ \\
\hline Gráficos & 1 & 0 & 0 & 0 & 0 & $\mathbf{1}$ & $\mathbf{1 \%}$ \\
\hline Gráficos e tabelas & 3 & 1 & 0 & 0 & 0 & $\mathbf{4}$ & $\mathbf{4 \%}$ \\
\hline Gráficos e quadros & 2 & 1 & 0 & 0 & 0 & $\mathbf{3}$ & $\mathbf{3 \%}$ \\
\hline Gráficos, quadros e tabelas & 4 & 0 & 1 & 0 & 1 & $\mathbf{6}$ & $\mathbf{6 \%}$ \\
\hline Quadros & 1 & 1 & 0 & 1 & 0 & $\mathbf{3}$ & $\mathbf{3 \%}$ \\
\hline Quadros e tabelas & 2 & 0 & 0 & 2 & 3 & $\mathbf{7}$ & $\mathbf{7 \%}$ \\
\hline Quadros e figuras & 2 & 0 & 0 & 2 & 0 & $\mathbf{4}$ & $\mathbf{4 \%}$ \\
\hline Tabelas & 0 & 1 & 0 & 1 & 0 & $\mathbf{2}$ & $\mathbf{2 \%}$ \\
\hline Fonte: Dados
\end{tabular}

Fonte: Dados da pesquisa.

$\mathrm{Na}$ Tabela 4, observa-se que dos recursos ilustrativos utilizados nas dissertações do programa de pós-graduação em ciências contábeis analisado, prevaleceu à utilização dos recursos ilustrativos do tipo figuras, quadros e tabelas que representam $42 \%$ dos recursos 
utilizados. Percebe-se ainda, que $18 \%$ das dissertações utilizaram figuras, gráficos, quadros e tabelas. As representações gráficas foram encontradas nas dissertações com abordagem qualitativa, quantitativa e qualitativo-quantitativa. Para Maroco (2003) a representação gráfica dos resultados tem por objetivo visualizar as características das variáveis em estudo, apresentando os resultados de forma simples e de fácil memorização. Os gráficos podem ser representados por histogramas de freqüências, gráficos de barras, gráficos circulares diagramas de dispersão e outros. Na sequência, a Tabela 5 aborda a quantidade de hipóteses presentes nas pesquisas analisadas por ano.

Tabela 5 - Quantidade de hipóteses utilizadas nas dissertações por ano

\begin{tabular}{c|c|c|c|c|c|c}
\hline \multirow{2}{*}{ Ano } & \multicolumn{7}{|c}{ Quantidade de hipóteses utilizadas nas dissertações } \\
\cline { 2 - 7 } & Uma & Duas & Três & Quatro & Cinco ou mais & Total \\
\hline $\mathbf{2 0 0 5}$ & 1 & 6 & 0 & 1 & 0 & $\mathbf{8}$ \\
\hline $\mathbf{2 0 0 6}$ & 0 & 2 & 0 & 0 & 0 & $\mathbf{2}$ \\
\hline $\mathbf{2 0 0 7}$ & 0 & 2 & 1 & 0 & 1 & $\mathbf{4}$ \\
\hline $\mathbf{2 0 0 8}$ & 1 & 2 & 0 & 2 & 2 & $\mathbf{7}$ \\
\hline $\mathbf{2 0 0 9}$ & 0 & 0 & 0 & 0 & 1 & $\mathbf{1}$ \\
\hline Total & $\mathbf{2}$ & $\mathbf{1 2}$ & $\mathbf{1}$ & $\mathbf{3}$ & $\mathbf{4}$ & $\mathbf{2 2}$ \\
\hline Percentuais & $\mathbf{9 \%}$ & $\mathbf{5 5 \%}$ & $\mathbf{5 \%}$ & $\mathbf{1 4 \%}$ & $\mathbf{1 8 \%}$ & $\mathbf{1 0 0 \%}$ \\
\hline
\end{tabular}

Fonte: dados da pesquisa

Por meio da Tabela 5, percebe-se das 102 dissertações analisadas no período de 2005 a 2009, apenas 22 continham hipóteses de pesquisas e destas 55\% apresentaram duas hipóteses de pesquisa. Percebeu-se ainda, que as 22 dissertações que apresentaram hipóteses enquadravam-se como pesquisas quantitativas e qualitativo-quantitativas. Estes resultados coadunam com os achados de Santos, Klann e Rausch (2010, p. 12) quando em sua pesquisa os autores inferem que "as hipóteses na maioria das vezes estão presentes em pesquisas quantitativas".

\subsection{Análise dos métodos estatísticos aplicados nas dissertações estudadas}

A seguir, apresenta-se análise dos métodos estatísticos mais utilizados nas dissertações do programa de pós-graduação em ciências contábeis da FURB, no período de 2005 a 2009 por meio da Tabela 6 .

Tabela 6 - Métodos estatísticos utilizados nas dissertações

\begin{tabular}{l|c|c|c|c|c|c|c}
\hline \multirow{2}{*}{$\begin{array}{c}\text { Métodos estatísticos aplicados } \\
\text { nas dissertações }\end{array}$} & \multicolumn{7}{c}{ Quantidade de dissertações defendidas por ano } \\
\cline { 2 - 8 } & $\mathbf{2 0 0 5}$ & $\mathbf{2 0 0 6}$ & $\mathbf{2 0 0 7}$ & $\mathbf{2 0 0 8}$ & $\mathbf{2 0 0 9}$ & \multicolumn{1}{c}{ Total } & $(\mathbf{\%})$ \\
\hline Estatística descritiva & 21 & 17 & 7 & 5 & 1 & $\mathbf{5 1}$ & $\mathbf{5 0 \%}$ \\
\hline Inferências estatísticas & 7 & 1 & 2 & 3 & 2 & $\mathbf{1 5}$ & $\mathbf{1 5 \%}$ \\
\hline Regressão e correlação & 2 & 3 & 2 & 3 & 0 & $\mathbf{1 0}$ & $\mathbf{1 0 \%}$ \\
\hline Análise multivariada dos dados & 3 & 7 & 2 & 4 & 2 & $\mathbf{1 8}$ & $\mathbf{1 8 \%}$ \\
\hline Abordagem multicritério & 1 & 1 & 0 & 0 & 1 & $\mathbf{3}$ & $\mathbf{3 \%}$ \\
\hline Modelagem matemática & 1 & 0 & 1 & 0 & 0 & $\mathbf{2}$ & $\mathbf{2 \%}$ \\
\hline Metaheurística & 0 & 1 & 1 & 0 & 0 & $\mathbf{2}$ & $\mathbf{2 \%}$ \\
\hline Data mining & 0 & 0 & 0 & 1 & 0 & $\mathbf{1}$ & $\mathbf{1 \%}$ \\
\hline Total & $\mathbf{3 5}$ & $\mathbf{3 0}$ & $\mathbf{1 5}$ & $\mathbf{1 6}$ & $\mathbf{6}$ & $\mathbf{1 0 2}$ & $\mathbf{1 0 0 \%}$ \\
\hline
\end{tabular}

Fonte: dados da pesquisa

As informações dispostas na Tabela 6 são provenientes da categorização dos métodos estatísticos proposta por Spegel (1993), Maroco (2003) e Corrar, Paulo e Dias Filho (2007). Em linhas gerais dentre os métodos estatísticos analisados, percebe-se a predominância da estatística descritiva presente em $50 \%$ das dissertações analisadas. Entretanto, os outros 50\% das dissertações utilizaram os métodos de análise multivariada dos dados (18\%), inferências 
estatísticas (15\%), regressão e correlação $(10 \%)$, abordagem multicritério $(3 \%)$, modelagem matemática e metaheurística (2\%) respectivamente e Data mining (1\%).

Neste contexto, em 2005 em torno de 21 dissertações fizeram uso da estatística descritiva em suas análises de dados, 14 dissertações adotaram outros métodos estatísticos, destas percebeu-se o predomínio das inferências estatísticas encontradas em sete dissertações. No ano de 2006, percebe-se novamente o predomínio da estatística descritiva frente aos demais métodos estatísticos, sendo encontrada em 17 dissertações. Cabe mencionar ainda, que das outras 13 dissertações que fizeram uso de outros métodos estatísticos a análise multivariada dos dados se sobressaiu, sendo encontrada em sete dissertações. Para tanto, observa-se em 2007 um crescimento dos outros métodos estatísticos em relação à estatística descritiva, percebe-se ainda uma pulverização dos métodos estatísticos nas dissertações analisadas, onde as inferências estatísticas, regressão e correlação foram encontradas em duas dissertações respectivamente e a análise multivariada dos dados, modelagem matemática, metaheurística foram encontradas em apenas uma dissertação respectivamente. Em 2008 das 16 dissertações aprovadas e defendidas, apenas cinco apresentaram estatística descritiva onde verifica-se o predomínio dos demais métodos como: análise multivariada de dados com quatro dissertações, inferências estatísticas e regressão e correlação com três dissertações respectivamente e uma dissertação com método Data mining. E por fim, no ano de 2009 das seis dissertações aprovadas e defendidas a estatística descritiva e abordagem multicritério estavam presentes em apenas uma dissertação respectivamente e as inferências estatísticas e análise multivariada dos dados em duas dissertações sucessivamente. Na sequência, a Figura 1 apresenta o comportamento dos métodos estatísticos utilizados nas dissertações analisados ao longo do período estudado realizando um comparativo entre a estatística descritiva e os outros métodos abordados neste estudo.

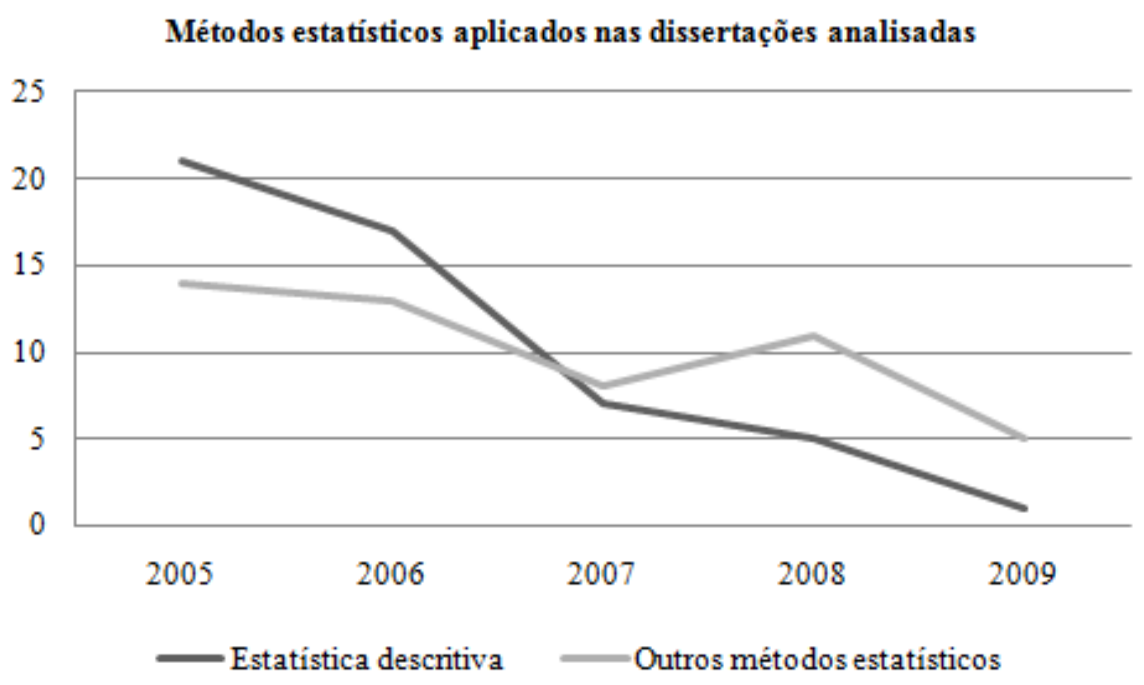

Figura 1 - Comportamento dos métodos estatísticos utilizados nas dissertações analisadas de 2005 a 2009 Fonte: Dados da pesquisa.

Com base nas informações expostas na Figura 1, observou-se que embora a estatística descritiva apresentar maior índice em relação aos demais métodos quantitativos nas dissertações do programa de pós-graduação em ciências contábeis analisado, percebe-se um declínio na utilização deste método ao longo do período estudado. Em contrapartida, denotase uma evolução na utilização dos outros métodos estatísticos durante o período investigado, tais como: análise multivariada dos dados, inferências estatísticas e regressão e correlação.

Neste sentido, infere-se que os métodos estatísticos encontrados nas dissertações do programa de pós-graduação em ciências contábeis da FURB, estão relacionados ao conceito de contabilometria que representa uma metodologia científica dos métodos quantitativos que 
envolvem a matemática, estatística bem como informática na área contábil (SILVA; CHACON; SANTOS, 2005). Ressalta-se ainda que, a informática pode estar relacionada ao uso de softwares como o Microsoft Excel que tem usa utilização disseminada nas pesquisas descritivas. Além dos outros softwares para estatísticos utilizados nos outros métodos estatísticos mencionados, tais como: SPSS, PLM, Statgraphics, LHstat dentre outros. A seguir, o Quadro 3 evidencia os métodos estatísticos encontrados nas dissertações e apresenta suas tipificações e/ou descrições.

\begin{tabular}{|l|l|}
\hline \multicolumn{1}{|c|}{ Métodos estatísticos } & \multicolumn{1}{|c|}{$\begin{array}{c}\text { Descrição e tipificação dos métodos estatísticos encontrados nas } \\
\text { dissertações provenientes do programa de pós-graduação analisado }\end{array}$} \\
\hline Estatística Descritiva & $\begin{array}{l}\text { Análise de conteúdo; Análise de frequência; Frequência absoluta; Frequência } \\
\text { relativa; Distribuição de frequência; Percentuais; média; mediana; moda; } \\
\text { desvio padrão; representação gráfica; coeficiente de variância mínimo; quartil; } \\
\text { matriz de Slack. }\end{array}$ \\
\hline Inferências Estatísticas & $\begin{array}{l}\text { Análise bivariada; percentagem sistêmica; teste t-Student; histograma; testes } \\
\text { estatísticos paramétricos; comparação de frequência; cálculo do grau de } \\
\text { associação (coeficiente de gama); comparação de médias com bicaudal direta e } \\
\text { utilização da tábua de TC (T Crítico); testes não-paramétricos Mann Whitney; } \\
\text { testes lambda de Will; Analisys of Variance. }\end{array}$ \\
\hline Regressão e Correlação & $\begin{array}{l}\text { Regressão e correlação; coeficiente de correlação ordinal de Kendall; } \\
\text { correlações; Frequências e medidas centrais; Regressão múltipla; Estatística de } \\
\text { regressão; erro padrão da regressão; Variável Dummie; R-quadrado. }\end{array}$ \\
\hline Análise Multivariada dos & $\begin{array}{l}\text { Análise de correspondência múltiplipla; clusters; análise de componentes } \\
\text { principais; análise discriminante múltipla; análise de conglomerados; análise } \\
\text { fatorial; agrupamentos hierárquicos, coeficiente de correlação Pearson; } \\
\text { ANOVA; conjuntos aproximativos; análise de agrupamentos hierárquicos. }\end{array}$ \\
\hline Abordagem multicritério & Análise envoltória dos dados; método análise hierárquica de processos. \\
\hline Modelagem matemática & Modelos matemáticos; fórmulas matemáticas \\
\hline Metaheurística & Conjuntos difusos. \\
\hline Data Mining & Teoria dos conjuntos aproximativos \\
\hline
\end{tabular}

Quadro 3 - Descrição dos métodos estatísticos utilizados nas dissertações

Fonte: Dados da pesquisa.

De acordo com o Quadro 3 a Estatística descritiva utilizada nas dissertações podem ser comparadas com a descrição de Maroco (2003) que as classifica como medidas de tendência central (média, mediana e moda); medidas de dispersão (coeficiente de variação; o intervalo de variação e a amplitude inter-quartis); medidas de assimetria e achatamento; medidas de associação e representação gráfica de resultados. Dessa forma, as dissertações que abordaram as inferências estatísticas, algumas apresentam tipificações citadas por Maroco (2003), tais como: distribuição do Qui-quadrado $\left(\mathrm{x}^{2}\right)$; distribuição t-Student, conforme comentado na seção de métodos quantitativos desse estudo. As dissertações que trabalham com regressão e correlação que conforme Spiegel (1993) envolvem correlação e regressão; correlação linear; medidas de correlação, linha de regressão de mínimos quadrados; erro padrão da estimativa; variação explicada e não-explicada; coeficiente de correlação; observações sobre o coeficiente de correlação; fórmula de covariância para o coeficiente de correlação linear; correlação ordinal; correlação de séries temporais; correlação de atributos; teoria amostral da correlação; teoria amostral da regressão; correlação múltipla. Para tanto, na análise multivariada de dados conforme mencionado por Corrar, Paulo e Dias Filho (2007) no qual descrevem que as técnicas de análise multivariada são: regressão múltipla; análise discriminante; regressão logística; análise fatorial; análise de conglomerados (clusters analysis); MDS (escalonamento multidimensional); redes neurais; lógica nebulosa aproximando-se das técnicas encontradas nas dissertações analisadas. $\mathrm{Na}$ abordagem multicritério as dissertações analisadas utilizam a análise envoltória dos dados mais conhecida como DEA e análise hierárquica de processos também denominada método AHP. E por fim, na modelagem matemática se elencou alguns 
modelos matemáticos e fórmulas matemáticas e a metaheurística contemplou a técnica de conjuntos difusos e o data mining utilizado na teoria dos conjuntos aproximativos.

\section{CONCLUSÕES}

A presente pesquisa teve por objetivo analisar os métodos estatísticos aplicados nas dissertações do programa de pós-graduação em ciências contábeis da Universidade Regional de Blumenau (FURB), no período de 2005 a 2009. Assim, na metodologia optou-se por uma pesquisa de cunho descritiva, realizada por meio de uma pesquisa documental com características bibliográficas onde a abordagem dos dados predominantemente quantitativa auxiliada por uma ficha padronizada. Cabe mencionar que a amostra compreendeu as 102 dissertações aprovadas e defendidas no programa de pós-graduação em ciências contábeis da FURB no período de 2005 a 2009, disponíveis no sítio do programa de pós-graduação coletadas durante o mês de maio de 2010. Resgata-se a seguir, a indagação que norteia a presente pesquisa: Quais são os métodos quantitativos mais recorrentes nas dissertações do programa de pós-graduação em ciências contábeis da Universidade Regional de Blumenau?

Em resposta a esta indagação, foi possível constatar que, durante o período analisado foram aprovadas e defendidas 102 dissertações no programa de pós-graduação analisado, onde $69 \%$ destas foram do gênero masculino e $31 \%$ feminino. Para tanto, no que se refere às linhas de pesquisas contempladas pelo programa observou-se que 69 dissertações pertencem à linha de pesquisa de Controle de Gestão e 33 dissertações são contempladas pela linha de pesquisa da área de Contabilidade Financeira.

No que tange as tipologias de pesquisa dos objetivos adotados nas dissertações analisadas, constatou-se que $43 \%$ apresentam natureza quantitativa, $32 \%$ qualitativa e $25 \%$ das dissertações demonstram natureza qualitativo-qualitativa. Infere-se ainda que, o predomínio da abordagem quantitativa nas dissertações que coadunam com os achados de Santos, Klann e Rausch (2010) quando afirmam que pesquisas dessa natureza, têm o intuito de quantificar fenômenos por meio de métodos estatísticos.

Quanto aos recursos ilustrativos aplicados nas dissertações analisadas, observou-se o predomínio das figuras, quadros e tabelas que representam $42 \%$ dos recursos empregados. Verificou-se ainda que, $18 \%$ das dissertações continham figuras, gráficos, quadros e tabelas. Estes achados corroboram com a afirmação de Maroco (2003) quando menciona tais recursos tem a função de permitir melhor visualização das variáveis em estudo, demonstrando-as de forma simples. Para tanto, com relação à quantificação das hipóteses de pesquisa utilizadas nas dissertações percebeu-se que 22 dissertações apresentaram hipóteses, sendo que $55 \%$ das dissertações utilizaram duas hipóteses. Estes achados corroboram com Santos, Klan e Rausch (2010) quando mencionam que as hipóteses são utilizadas nas pesquisas quantitativas para favorecer a interpretação dos dados.

Em relação aos métodos estatísticos aplicados nas dissertações do programa de pósgraduação em ciências contábeis da FURB, a estatística descritiva foi o método estatístico predominante em 51 dissertações analisadas que correspondem a 50\% da amostra. Para tanto, nas outras 51 dissertações os métodos estatísticos mais recorrentes foram: análise multivariada dos dados $(18 \%)$, inferências estatísticas $(15 \%)$, regressão e correlação $(10 \%)$, abordagem multicritério (3\%), modelagem matemática e metaheurística com $2 \%$ respectivamente e o Data mining presente em apenas $1 \%$ das dissertações estudadas. Cabe mencionar ainda que, nos últimos anos percebeu-se uma evolução na utilização dos métodos estatísticos, tais como: análise multivariada dos dados, inferências estatísticas e regressão e correlação quanto comparado com a estatística descritiva empregada nas dissertações inicialmente. Neste sentido, infere-se que o conceito de contabilometria está presente nas dissertações analisadas quando faz uso de uma metodologia científica dos métodos 
quantitativos que envolvem a matemática, estatística e informática nas pesquisas da área contábil, conforme afirmam Silva, Chacon e Santos (2005).

Neste contexto, percebe-se que os métodos estatísticos vêm sendo utilizados com maior freqüência neste programa de pós-graduação em ciências contábeis que tem por intuito incentivar as pesquisas quantitativas. Assim, infere-se que estas pesquisas tendem a desenvolver e contribuir para a interpretação dos fenômenos sociais investigados a fim de proporcionar uma evolução científica. Frente às limitações da presente pesquisa, sugere-se para futuras pesquisas realizar esta pesquisa futuramente a fim de traçar a evolução dos métodos estatísticos aplicados nas dissertações dos programas de pós-graduação em ciências contábeis da FURB, além de reaplicar o estudo em outros programas de pós-graduação em ciências contábeis brasileiros.

\section{REFERÊNCIAS}

BEUREN, I. M. (Org). Como elaborar trabalhos monográficos em contabilidade: teoria e prática. São Paulo: Atlas, 2008.

BOUDON, R. Os métodos em sociologia. São Paulo: Ática, 1989.

CANECA, R. L.; VASCONCELOS, M. T. C. Um estudo sobre a importância da aplicação dos métodos quantitativos à contabilidade. In: Congreso Internacional de Costos, 10., 2007, França. Anais... França: CIC, 2007. CD-ROM.

CAPES - COORDENAÇÃO DE APERFEIÇOAMENTO DE PESSOAL DE NÍVEL SUPERIOR. Programas de pós-graduação recomendados. Disponível em: http://www.capes.gov.br. Acesso em: Abril 2010.

CERVO, A. L.; BERVIAN, P. A. Metodologia científica. $4^{\text {a }}$ ed. São Paulo: Makron Books, 1996.

CORRAR, L. J.; PAULO, E.; DIAS FILHO, J. M. Análise multivariada: para os cursos de administração, ciências contábeis e economia. São Paulo: Atlas, 2007.

FASSINA, P. H. Análise do endividamento e liquidez de empresas por meio de um sistema especialista difuso. 2006. 201f. Dissertação (Mestrado em Ciências Contábeis). Departamento de Pós Graduação em Ciências Contábeis da Universidade Regional de Blumenau. Blumenau, 2006.

FIGUEIREDO, S.; MOURA, H. A utilização dos Métodos Quantitativos pela Contabilidade. Revista Brasileira de Contabilidade, Brasília, ano 30, n.127, p. 51-61, jan./fev. 2001.

FURB - UNIVERSIDADE REGIONAL DE BLUMENAU. Dissertações em ciências contábeis. Disponível em: http:/wwww.furb.br. Acesso em Março de 2010.

GALlON, A. V.; SOUZA, F. C.; ROVER, S.; ENSSLIN, S. R. Análise das IES da área de Ciências Contábeis e de seus pesquisadores por meio de sua produção científica. In: Congresso Brasileiro de Contabilidade, 18., 2008, Gramado. Anais... Gramado: CRC, 2008. CD-ROM.

GRILHO JÚNIOR, T. F.; XAVIER, G. B.; CLAUDINO NETO, S.; CLERICUZI, A. Z. Proposta de um framework aplicando técnicas de data mining para o controle externo no tribunal de contas do estado da Paraíba. In: International Conference on Information Systems and Technology Management, 6., 2009, São Paulo. Anais... São Paulo: CONTECSI, 2009. CD-ROM. 
GALVINO, C. C. T.; SOUZA, O. D. Bibliometria e biblioteca universitária: um estudo analítico das citações das dissertações de mestrado do Programa de Pós-Graduação em Psicologia Social (2004-2006) da Universidade Federal da Paraíba. In: Seminário Nacional de bibliotecas universitárias, 15., 2008. São Paulo. Anais ... São Paulo: SBBU, 2008. CD-ROM.

LYRA, R. L. W. C. Análise hierárquica dos indicadores contábeis sob óptica do desempenho empresarial. 2008. 184 p. Dissertação. (Mestrado em Ciências Contábeis). Departamento de Pós Graduação em Ciências Contábeis da Universidade Regional de Blumenau. Blumenau, 2008.

MARTINS, G. A.; THEÓPHILO, C. R. Metodologia da investigação científica para ciências sociais aplicadas. São Paulo: Atlas, 2007.

MAROCO, J. Análise estatística: com utilização do SPSS. 2. ed. Lisboa: Edições Sílabo. 2003.

MATSUMOTO, A. S.; PEREIRA, S. E.; NASCIMENTO, G. S. A utilização da Contabilometria e a agregação de valor à informação contábil. In: Congresso USP de Contabilidade e Controladoria, 6., 2006. Anais... São Paulo: FEA/USP, 2006. CD-ROM.

NOSSA, V.; GARCIA, S. Contabilometria: Uma proposta de ensino para o curso de Ciências Contábeis. In: CONVENÇÃO DOS CONTABILISTAS, 2002, Espírito Santo. Anais... Espírito Santo: CONVENÇÃO DOS CONTABILISTAS, 2002.

RAUPP, F. M.; BEUREN, I. M. Metodologia da Pesquisa Aplicável as Ciências Sociais. In: BEUREN, Ilse Maria (Org.). Como Elaborar Trabalhos Monográficos em Contabilidade: Teoria e Prática. São Paulo: Atlas, 2008.

RICCIO, E. L.; SAKATA, M.; CARASTAN, J. T.; Accounting research in brazilian universities: 1962-1999. Caderno de Estudos. Fundação Instituto de Pesquisas Contábeis, Atuariais e Financeiras. São Paulo, v. 11, n. 22, p. 35-44, set.-dez., 1999.

RIBEIRO FILHO, J. F.; LOPES, J. E. G.; SOUZA, I. G. A.; PEDERNEIRAS, M. M. M. Uma Análise das Abordagens Epistemológicas e Metodológicas da Pesquisa Contábil do Programa do Mestrado Multiinstitucional em Ciências Contábeis. Contabilidade Vista \& Revista, v. 18, n.1, 2007.

SANTOS, V.; KLANN, R. C.; RAUSCH, R. B. Perfil das dissertações dos programas de pósgraduação em ciências contábeis da FEA/USP e da FURB. In: Associação Nacional dos Programas de Pós-graduação em Ciências Contábeis, 4., 2010, Natal. Anais... Natal: ANPCONT, 2010. CD-ROM. p.1-15.

SILVA, M. C.; CHACON, M. J. M.; SANTOS, J. O que é Contabilometria? Revista Pensar Contábil, v.7, n.27, p.40-43, fev./abr. 2005.

SPIEGEL, M. R. Estatística. 3. ed. São Paulo: Makron Books, 1993.

THEÓPHILO, C. R.; IUDÍCIBUS, S. Uma Análise Crítico-Epistemológica da Produção Científica em Contabilidade no Brasil. In: Encontro da Associação Nacional de PósGraduação e Pesquisa em Administração, 29., Brasília. Anais... Rio de Janeiro: ENANPAD, 2005. CD-ROM. 